\title{
New Invagination Procedure for Pancreaticojejunostomy Using Only Four Sutures: Reply
}

\author{
Yoshitsugu Nakanishi - Masanori Ohara • \\ Misa Noguchi · Hiromitsu Domen • \\ Kazuteru Komuro • Satoshi Hirano
}

Published online: 26 February 2013

(C) Société Internationale de Chirurgie 2013

We thank you for your comments regarding our article entitled "New invagination procedure for pancreaticojejunostomy using only four sutures" [1].

You state that conventional pancreatic anastomosis (PA) is still a good choice and do not believe that our procedure offers a better option than conventional pancreaticojejunostomy (PJ). The reasons we believe it is a good choice are that many studies, including three randomized controlled trials (RCTs), have failed to identify the "best" PA technique. Patient factors, including the texture of the pancreas (hard or soft) and obesity, are related to the occurrence of a pancreatic fistula (PF). Thus, you insist that the occurrence of a PF is largely unaffected by the type of PA but, instead, is determined mainly by patient factors.

In fact, all surgeons are worried about outcomes after performing a PA because no previous procedures have been able to prevent PFs. We should thus make an effort to devise better procedures that many surgeons could perform simply and reliably. Our goal in devising this surgical technique was to establish a new procedure for PA that could be easily performed by any surgeon without risk of a $\mathrm{PF}$ for the patient.

As mentioned in our report, our new procedure is simple and economical compared to a number of conventional

\footnotetext{
Y. Nakanishi $(\bowtie) \cdot$ M. Ohara $\cdot$ M. Noguchi $\cdot$ H. Domen

K. Komuro

Department of Surgery, National Hospital Organization,

Hakodate Hospital, 18-16 Kawahara-cho,

Hakodate 0041-8512, Japan

e-mail: y.nakanishi@mac.com

Y. Nakanishi $\cdot$ M. Noguchi $\cdot$ H. Domen $\cdot$ S. Hirano

Department of Gastroenterological Surgery II, Hokkaido

University Graduate School of Medicine, North 15,

West 7, Kita-ku, Sapporo 060-8638, Japan
}

procedures performed worldwide at this time. Moreover, we suggested that minimizing the number of stitches in the pancreatic parenchyma might avoid leakage of pancreatic juice from puncture holes into the pancreas and from crushing the pancreatic parenchyma. The result of $0 \%$ for grade $\mathrm{B}$ or $\mathrm{C}$ PFs is most satisfactory, but it also indicates that the procedure has to be refined. A review of the literature revealed that the PF formation rate with conventional pancreaticojejunostomy ranges between 7 and $28 \%$, as you mentioned in your letter. Importantly, this rate includes PF of grades B and C, which sometimes results in patients entering a critical condition. PF grade $\mathrm{A}$ is defined as minor leakage with no apparent physical difficulty and, from a clinical perspective, clearly differs from grade B or C.

Because, as our article indicated, the findings were only preliminary and gathered retrospectively for a small number of patients with some variation in pancreatic texture, we never concluded that our procedure represents the optimal procedure for PA. We therefore plan to undertake a randomized study to compare this new procedure with conventional techniques.

In summary, at this time, each pancreatic surgeon should perform the procedures for PA to which he or she is most accustomed. Also, however, there is a need to continue innovating so new techniques can reduce the morbidity associated with surgery while minimizing the risk of a PF.

\section{Reference}

1. Nakanishi Y, Ohara M, Noguchi M et al (2012) New invagination procedure for pancreaticojejunostomy using only four sutures. World J Surg 36:892-897. doi:10.1007/s00268-012-1441-3 